\title{
Discovery of a General Method of Solving the Schrödinger and Dirac Equations That Opens a Way to Accurately Predictive Quantum Chemistry
}

\author{
HIROSHI NAKATSUJI* \\ Quantum Chemistry Research Institute and JST-CREST, Kyodai Katsura Venture \\ Plaza 107, Goryo Oohara 1-36, Nishikyo-ku, Kyoto 615-8245, Japan
}

RECEIVED ON DECEMBER 30, 2011

\section{CONSPECTUS}

J

ust as Newtonian law governs classical physics,

the Schrödinger equation (SE) and the relativistic Dirac equation (DE) rule the world of chemistry. So, if we can solve these equations accurately, we can use computation to predict chemistry precisely. However, for approximately 80 years after the discovery of these equations, chemists believed that they could not solve SE and DE for atoms and molecules that included many electrons. This Account reviews ideas developed over the past decade to further the goal of predictive quantum chemistry.

Between 2000 and 2005, I discovered a general method of solving the SE and DE accurately. As a first inspiration, I formulated the structure of the exact wave function of the SE in a compact mathematical form. The explicit inclusion of the exact wave function's structure within the variational space allows for the calculation of the exact wave function as a solution of the variational method. Although this process sounds almost impossible, it is indeed possible, and I have published several formulations and applied them to solve the full configuration interaction $(\mathrm{Cl})$ with a very small number of variables. However, when I examined analytical solutions for atoms and molecules, the Hamiltonian integrals in their secular equations diverged. This singularity problem occurred in all atoms and molecules because it originates from the singularity of the Coulomb potential in their Hamiltonians. To overcome this problem, I first introduced the inverse $S E$ and then the scaled $S E$. The latter simpler idea led to immediate and surprisingly accurate solution for the SEs of the hydrogen atom, helium atom, and hydrogen molecule.

The free complement (FC) method, also called the free iterative CI (free ICI) method, was efficient for solving the SEs. In the FC method, the basis functions that span the exact wave function are produced by the Hamiltonian of the system and the zeroth-order wave function. These basis functions are called complement functions because they are the elements of the complete functions for the system under consideration. We extended this idea to solve the relativistic DE and applied it to the hydrogen and helium atoms, without observing any problems such as variational collapse. Thereafter, we obtained very accurate solutions of the SE for the ground and excited states of the Born-Oppenheimer (BO) and non-BO states of very small systems like $\mathrm{He}, \mathrm{H}_{2}{ }^{+}, \mathrm{H}_{2}$, and their analogues. For larger systems, however, the overlap and Hamiltonian integrals over the complement functions are not always known mathematically (integration difficulty); therefore we formulated the Iocal SE (LSE) method as an integral-free method. Without any integration, the LSE method gave fairly accurate energies and wave functions for small atoms and molecules. We also calculated continuous potential curves of the ground and excited states of small diatomic molecules by introducing the transferable local sampling method. Although the FC-LSE method is simple, the achievement of chemical accuracy in the absolute energy of larger systems remains time-consuming. The development of more efficient methods for the calculations of ordinary molecules would allow researchers to make these calculations more easily. 


\section{Introduction}

The Schrödinger equation (SE) and the relativistic Dirac equation (DE) are the basic equations that govern the world of atoms and molecules, ${ }^{1}$ just as the Newton's and Einstein's laws govern the macroscopic and cosmic world. ${ }^{2}$ In the latter world, the basic principles are able to provide very accurate predictions that enable applications such as interplanetary journeys to occur. However, in the former world, such accurate predictions are limited only to the hydrogen atom and some simple oneelectron systems. For many-electron atoms and molecules that are daily subjects in chemistry, even modern quantum chemistry cannot provide predictions as accurate as those in the Newtonian world. The reason for this is simple. The basic equations in classical mechanics can be solved to their intrinsic accuracies, but in the quantum world, the SE and DE have not been solved in their limiting accuracies: they are solved only approximately. People thought for many years, and after many trials, that solving the SE and DE was essentially impossible. This was so for about 80 years after the discovery of the SE.

Modern quantum chemistry is much developed and provides useful tools for understanding ${ }^{1}$ the underlying nature of chemical phenomena. However, even the advanced theories encounter difficulties when applied to bond-fission processes. ${ }^{3}$ However, if a general and accurate method of solving the $S E$ and $D E$ is established, we will be able to enjoy truly predictive quantum chemistry. Some years ago, the author discovered a general method of solving the $\mathrm{SE}$ and $\mathrm{DE},{ }^{4-10}$ and this method is now being developed in our Institute to realize the above dream. The purpose of this Account is to provide an expository review of the basic ideas and their applications to calculate the full configuration interaction (CI) and analytical solutions of the SE of atoms and molecules. Only minimum possible formulas and mathematics will be used.

\section{How Accurate Are the SE and DE?}

The hydrogen atom is the only example for which the SE and DE are exactly solvable. The exact energy of the SE is

$$
E(n)=-\mu Z^{2} /\left(2 n^{2}\right)
$$

where $Z$ is the nuclear charge, $n$ is the principal quantum number, and $\mu$ is the reduced mass. Similarly, the exact energy of the $\mathrm{DE}$ is given by ${ }^{11}$

$$
E\left(n^{\prime}, j\right)=\left(\frac{1}{\sqrt{1+\frac{Z^{2} \alpha^{2}}{\left(n^{\prime}+\sqrt{\left.(j+1 / 2)^{2}-Z^{2} \alpha^{2}\right)^{2}}\right.}}}-1\right) \mu
$$

where $n^{\prime}$ is similar to $n$ above, $j$ is the total angular momentum quantum number, and $\alpha=1 / 137.03599$ is the fine structure constant. We used the atomic unit in this Account (for energy, $1 \mathrm{au}=27.21134 \mathrm{eV}, 627.5096$ $\mathrm{kcal} / \mathrm{mol}$, or $219474.6315 \mathrm{~cm}^{-1}$ ). For the hydrogen atom, the experimental ground-state energy ${ }^{12}$ is -0.499733191 , the SE energy is -0.499727840 , and the DE energy is -0.49973449 . We can calculate the excitation energy for the excitation from $n=1$ to $n=2$. The experimental values are 0.374799317 for $2 s(1 / 2)$, 0.374799158 for $2 p(1 / 2)$, and 0.374800821 for $2 p(3 / 2)$, where small differences are observed for the $2 s$ and $2 p$ states. The value in parentheses is the $j$ value. The SE gives only a single value of 0.374795880 for these three different states. The DE gives a value of 0.374800452 for the $2 s(1 / 2)$ and $2 p(1 / 2)$ states and 0.374802115 for the $2 p(3 / 2)$ state. The DE cannot explain the small difference between the $2 s(1 / 2)$ and $2 p(1 / 2)$ states. This difference is called the Lamb shift and is explained by quantum electrodynamic (QED) theory, ${ }^{11}$ which is beyond the scope of the present Account.

The reader has no doubt been very impressed with the high accuracy of the energies predicted by the SE and DE. The SE predicts the energy to four-digit accuracy and the DE to five digits. Certainly, the DE gives a higher accuracy than the $\mathrm{SE}$, but the $\mathrm{SE}$ is already highly accurate for the hydrogen atom. This is true for the first row elements in the Periodic Table. For heavier elements, the relativistic effects expressed by the DE become more important. Therefore, if we have a theory that enables us to solve the SE and DE to a high accuracy and if we can apply it to atoms and molecules that appear daily in chemistry, then our science will be highly elevated to be truly predictive for chemical science. In this case, computer simulations would become a complementary methodology that would compete with experiments. Is this just a dream? Is there any way to realize this dream? We are trying to realize this dream.

\section{The Schrödinger and Dirac Equations}

What are the SE and DE? They have very simple and similar forms. The SE is written as

$$
H \psi=E \psi
$$

where the Hamiltonian is given by

$$
H=-\sum_{i} \frac{1}{2} \Delta_{i}-\sum_{i} \sum_{A} \frac{Z_{A}}{r_{A i}}+\sum_{i>j} \frac{1}{r_{i j}}+\sum_{A>B} \frac{Z_{A} Z_{B}}{R_{A B}}
$$


in ordinary notation. The SE is a second-order differential equation. This differential equation can be solved analytically for the hydrogen atom, as explained above. However, for more-than-two electron systems, there is no direct analytical way to solve their differential equations. In our general method of solving the SE, we do not try to solve the "differential" equation. It is noted here that the differentiation is always possible for the analytical functions. In contrast, integration is not always "possible" for all analytical functions, even though one may know intuitively the existence of the integral values. It is fortunate that the $\mathrm{SE}$ does not include any integration in its expression.

The DE is written similarly to the SE as

$$
\mathbf{H} \Psi=E \Psi
$$

where the Hamiltonian, $\mathbf{H}$, is not a scalar operator but is a matrix operator with the dimension of $N^{4}$, with $N$ being the number of electrons of the system. Similarly, $\Psi$ is a vector function with the same dimension. This Account is mainly focused on the SE, so we will not give the explicit form of the Hamiltonian of the DE. However, we note that what we call DE here is actually the Dirac-Coulomb equation, which is a generalization of the one-electron Dirac equation ${ }^{11}$ to the many-electron case. Because of the similarity between the SE and DE, the method of solving the $\mathrm{SE}$ can be applied to the $\mathrm{DE}^{9}$ with a small modification. Finally, we note that we specify the true solutions of the SE and DE by using the word "exact".

\section{Variational Principle}

Probably the most frequently used principle in computational chemistry is the variational principle (VP),

$$
\delta E[\psi]=0 .
$$

Suppose that we try to solve the SE of a given system. We use $\psi$ as the candidate for a solution. It includes some variable parameters within an assumed functional form. An important merit of the VP is that it always provides the optimal values for such parameters. However, it is seldom to obtain the solution of the SE using this method, because it is very difficult to predict the exact functional form of a many-electron system by intuition alone. Even so, this principle gives us the best possible wave function within the assumed functional form.

If we can prepare a trial function that surely includes the exact wave function within its functional form, the VP method gives the exact wave function as its solution. Most people would probably think that this is not possible. However, it is both possible and easy, as shown below. We shall designate such a functional form as having an exact structure.

\section{Exact Structure}

In a series of papers starting from 2000 , the author has published several ideas under the title of "the structure of the exact wave function" ${ }^{4,5,13-15}$ The details are given in the references, but what we need here is simple. Let us compare three very important fundamental formulas. The first is the SE itself,

$$
(H-E) \psi=0
$$

which defines the exact wave function and its energy. The second formula is the VP, which is now written as

$$
\langle\psi|H-E| \delta \psi\rangle=0
$$

The third formula is what we call an $\mathrm{H}$-square equation,

$$
\left\langle\psi\left|(H-E)^{2}\right| \psi\right\rangle=0
$$

The $\mathrm{H}$-square equation is unique in that it is equivalent to the SE. Only the solution of the SE can satisfy this equation. The proof is simple and has been given previously.'

Now let us compare eqs 8 and 9 . Then, we can easily reach the following theorem.

Theorem. Let $\psi$ be a function that includes a parameter $C$ and satisfies the relationship

$$
\delta \psi=(H-E) \psi \cdot \delta C
$$

Then this $\psi$ has an exact structure, because its variational solution satisfies the $\mathrm{H}$-square equation and therefore is exact.

Proof. Because $\delta \psi$ is given by eq 10 , we can substitute it into the VP, eq 8, and obtain $\left\langle\psi\left|(H-E)^{2}\right| \psi\right\rangle \cdot \delta C=0$. Since this equation must hold for any $\delta C$, we obtain eq 9 . Namely, when $\psi$ satisfies eq 10, its VP solution satisfies the $\mathrm{H}$-square equation at the same time, and therefore it is exact (end of proof).

Thus, when we prepare our wave function to satisfy eq 10, its variational solution is guaranteed to be exact. It is very important to use a wave function that has an exact structure to formulate the method of solving the SE.

\section{Simplest Iterative Complement Method}

What $\psi$ satisfies eq 10 ? There are several possibilities. ${ }^{8}$ Among these, a simple expression is given by the following recursion formula: ${ }^{4,5}$

$$
\psi_{n+1}=\left[1+C_{n}\left(H-E_{n}\right)\right] \psi_{n}
$$




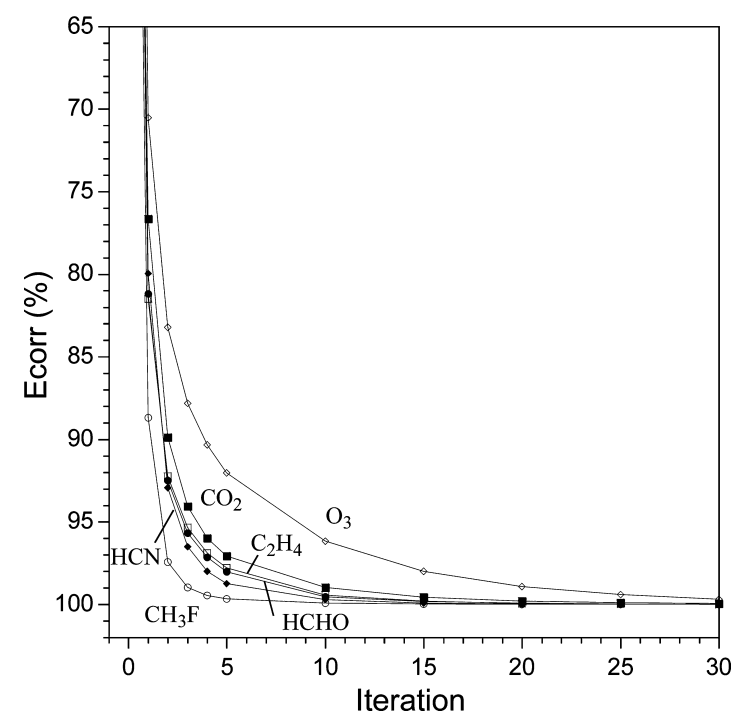

FIGURE 1. Convergence process of the SIC calculations to the full-CI energy using the ordinary Hamiltonian matrix of the minimal STO-6G basis

where $n$ is the iteration number, $C_{n}$ is one parameter in each iteration, and $E_{n}=\left\langle\psi_{n}|H| \psi_{n}\right\rangle$. Because this is the simplest form among what we call iterative complement (IC) methods, we shall denote it as the simplest IC (SIC) method. It is easy to prove that a SIC wave function becomes exact at convergence.' Applying the variational principle to eq 11 , we obtain a very simple $2 \times 2$ secular equation, and by solving it successively, we are able to obtain an exact solution of the SE at convergence.

\section{Full Configuration Interaction}

When we solve the SE within a given basis set, the solution is the so-called full $\mathrm{Cl}$ (configuration interaction). This is usually obtained after the diagonalization of a very large Hamiltonian matrix, which makes this method difficult to perform for large molecules with a very good basis set.

Insofar as the SIC equation is correct, we should be able to calculate the full-Cl solution using the SIC method starting from an approximate function, such as the Hartree-Fock, for $\psi_{0}$. This is performed by replacing the Hamiltonian operator in eq 11 with the Hamiltonian matrix over the full-Cl configurations. Figure 1 shows the results for several molecules using minimal basis set. ${ }^{16,17}$ Certainly, all the calculations converged to the full- $\mathrm{Cl}$ energies after the iterations were performed. For $\mathrm{HNCO}$, the full-Cl calculation was performed by the diagonalization of the Hamiltonian matrix with a dimension of 216247 , while the same result was obtained using the SIC method after 68 iterations of a twodimensional diagonalization. However, the convergence

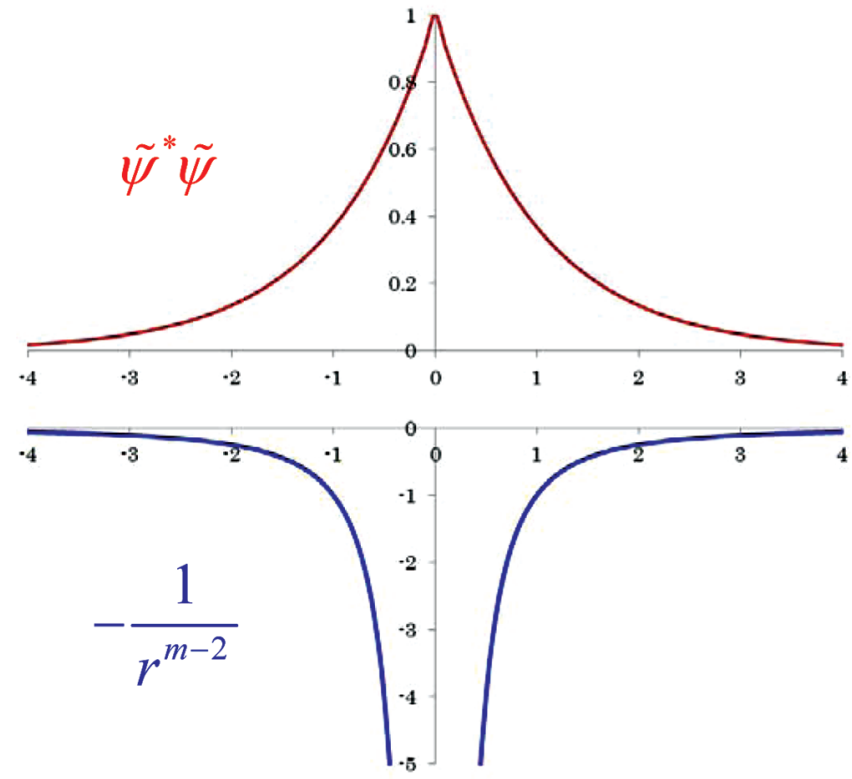

FIGURE 2. Illustrative proof of the singularity of the Hamiltonian integral expressed by eq 12 .

rate was very slow by the reason that will be explained later. $^{18}$

\section{Singularity Problem}

The full $\mathrm{Cl}$ is best only within a given basis set. When we improve the basis set, the dimension of the full $\mathrm{Cl}$ increases rapidly and a solution becomes impossible. Therefore, a better way is to solve the SE analytically. However, when we perform the SIC calculations analytically, we encounter a significant obstacle that we call the singularity problem. ${ }^{6-8}$ This is caused by the Coulomb potential that is included in the Hamiltonians of atoms and molecules. The nuclearelectron attraction potential and the electron-electron repulsion potential become infinite when two particles meet. For this reason, the integral of the Hamiltonian over an approximate wave function, $\tilde{\psi}$, diverges to plus or minus infinity when the integer $m$ has a value of $m \geq 3$, as follows.

$$
\left\langle\tilde{\psi}\left|H^{m}\right| \tilde{\psi}\right\rangle= \pm \infty \quad(m \geq 3)
$$

The proof is easy, as is shown in Figure 2. The upper part shows the quantity $\tilde{\psi}^{*} \tilde{\psi}$, which is always finite. The lower part shows the $m$ th power of the Coulomb potential multiplied by the Jacobian with order $r^{2}$. Near to the origin, the lower factor becomes minus infinity when $m \geq 3$, and therefore the integral diverges to infinity. This singularity problem is actually a significant obstacle. For example, in SIC calculations (eq 11), the $n$th order function includes the term $H^{n} \psi_{0}$. Therefore, the Hamiltonian matrix of this term 


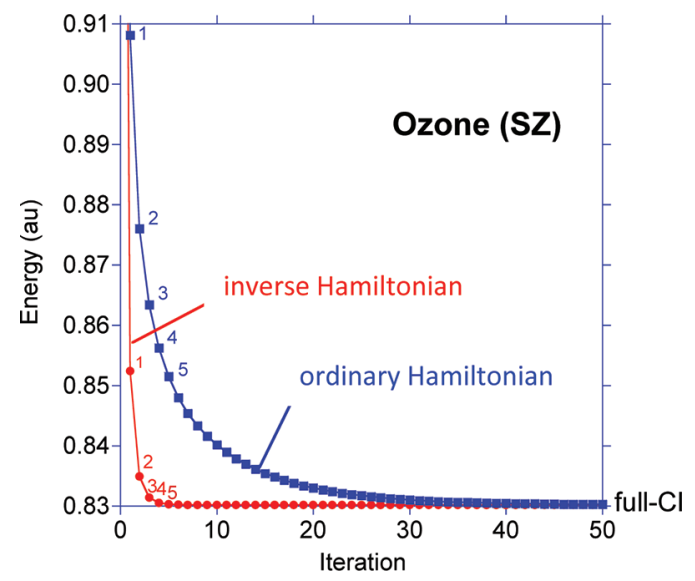

FIGURE 3. Convergence process of the SIC calculations of ozone to the full-Cl energy using the ordinary (blue, same as in Figure 1) and inverse (red) Hamiltonian matrices of the minimal STO-6G basis. ${ }^{18}$

includes $H^{2 n+1}$, and so diverges even when $n=1$. Therefore, the analytical calculations break down.

\section{Inverse Schrödinger Equation}

The author proposed two different ways to avoid the singularity problem. The first was to introduce the inverse Schrödinger equation (ISE), ${ }^{6}$

$$
H^{-1} \psi=E^{-1} \psi
$$

where $H^{-1}$ is the inverse Hamiltonian and $E^{-1}$ is the inverse of the energy $E$. This equation is equivalent to the original $\mathrm{SE}^{6}$ Even infinite values become zero when we introduce the inverse Hamiltonian.

In the SIC calculations of the full $\mathrm{Cl}$ shown in Figure 1, we multiplied the full-Cl Hamiltonian matrix many times. This could be done simply owing to the crudeness of the basis set: the STO-6G set cannot be a complete set. But, with the analytic Hamiltonian, this is, in principle, not possible because of the singularity problem. Therefore, SIC calculations with ordinary Hamiltonians are problematic, which is a reason for the slow convergence observed in Figure $1 .{ }^{18}$ When we take the inverse of the full-Cl Hamiltonian matrix, then it is the matrix of the inverse Hamiltonian, so what happens when we perform SIC calculations using the inverse Hamiltonian matrix?

Figure 3 shows a comparison of SIC calculations of ozone using ordinary (blue) and inverse (red) Hamiltonian matrices. ${ }^{18}$ When we use the inverse Hamiltonian, which is free from the singularity problem, the convergence speed is rapid. ${ }^{18}$ We note that a similar rapid convergence was also seen when we used the general singles and doubles (GSD) case with the IC method. ${ }^{19}$ However, in the analytical case, explicit expression of the inverse Hamiltonian was difficult to achieve.

\section{Scaled Schrödinger Equation}

The author then introduced the scaled Schrödinger equation $(\mathrm{SSE})^{7}$ to avoid the singularity problem,

$$
g(H-E) \psi=0
$$

where $g$, the scaling function, is a function of the electron coordinates. It has a positive value everywhere but can be zero at a singular point, $r_{0}$, and satisfies $\lim _{r \rightarrow r_{0}} g V=a$, with $a$ being a finite value and $V$ the Coulomb potential. This condition is necessary because $g$ must not eliminate the information at a singular point: the existence of a singularity may cause a problem, but it has a physical origin. The SSE is equivalent to the SE, because we can define $g^{-1}$. We often use a $g$ function with the form

$$
g=\sum r_{A i}+\sum r_{i j}
$$

After the introduction of the SSE, we can redefine the SIC method with

$$
\psi_{n+1}=\left[1+C_{n} g\left(H-E_{n}\right)\right] \psi_{n}
$$

which differs from the original form (eq 11) only in the presence of the $g$ term in front of the $H$ term, and this SIC gives, without encountering the singularity problem, the exact wave function at convergence. ${ }^{7}$ Note here that when we use the $g$ operator given by eq 15 , some diverging functions are also generated, but they should be discarded because the wave function must be integratable and finite. When we introduce a local $g$ operator, ${ }^{20}$ we can minimize such diverging functions, and the wave function takes a physically compact form.

\section{Free Complement Method}

When the SIC iterations are performed $n$ times, then the right-hand side of eq 16 becomes a sum of the analytical functions having different coefficients. We select all the independent functions $\left\{\phi_{i}\right\}\left(i=1, \ldots, M_{n}\right)$, give each an independent coefficient $\left\{\boldsymbol{c}_{i}\right\}$ and write $\psi_{n+1}$ as

$$
\psi_{n+1}=\sum_{i}^{M_{n}} c_{i} \phi_{i}
$$

which is referred to a free complement (FC) method. ${ }^{7}$ The function $\phi_{i}$ is referred to as a "complement" function, because for sufficiently large $n$ the functions $\left\{\phi_{i}\right\}$ span the exact wave function: each $\phi_{i}$ is an element of the complete function. The FC method was originally referred to as the free iterative complement (configuration) 


\section{Free Complement (FC) Method for Solving the Schrödinger Equation}

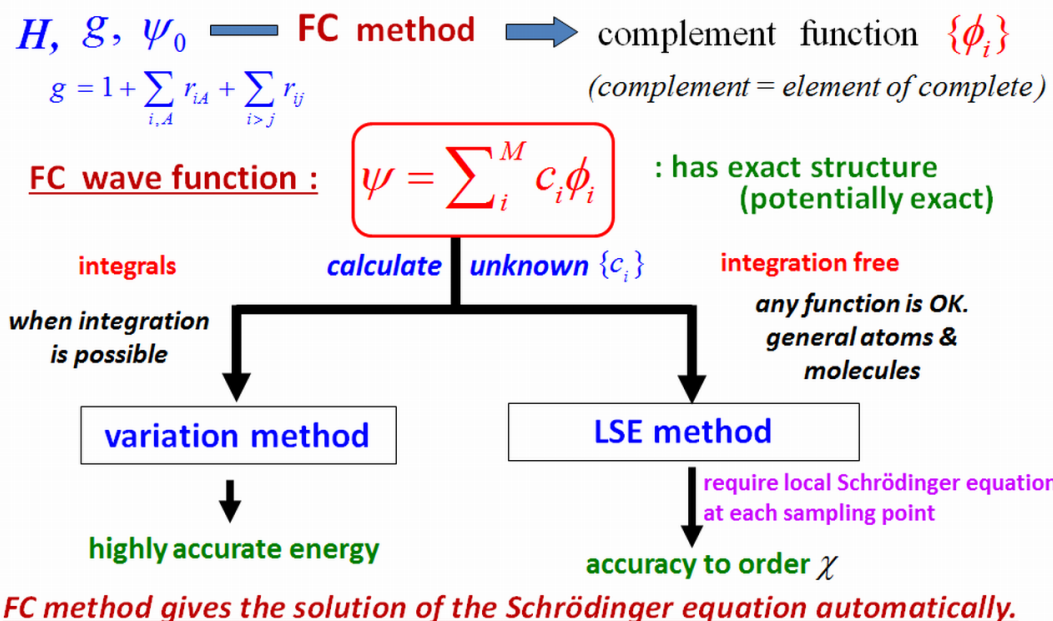

1) Hamiltonian includes all the information of the system.

2) Hamiltonian paves the way to its-own exact wave function.

3) When Hamiltonian is available, the analytic solution is automatic.

FIGURE 4. Flowchart of the FC method for solving the SE.

interaction (free ICl) method. ${ }^{7}$ The word "free" arises from the fact that we make all the independent analytical functions free in moving from eq 16 to eq 17. Using the FC method, the convergence to the exact wave function is accelerated more than in the case of the IC method. ${ }^{7,8}$

Figure 4 illustrates the use of the FC method for solving the SE. The FC method describes the exact wave function using a linear sum of the analytical complement functions that are produced by applying the Hamiltonian to $\psi_{0}$ (the $g$ function is also produced from the Hamiltonian). Thus, in FC formalism, the Hamiltonian that defines the system produces the basis (complement) functions for describing its exact wave function. The functional form of the complement function is determined by the form of the initial function, $\psi_{0}$. Slater-type complement functions are generated from the Slater-type $\psi_{0}$, and Gaussian-type complement functions are generated from the Gaussian-type $\psi_{0}{ }^{21}$ We believe that in this way we obtain the best possible basis functions for describing the system. We have no basis-set nightmares in our formalism. This is true even for the atoms and molecules in an external field such as a magnetic field. ${ }^{22,23}$ The FC wave function has an exact structure at $M$ sufficiently large and so is a potentially exact wave function. Therefore, the coefficients, $\left\{c_{i}\right\}$, should be determined using only the necessary conditions for the SE.

Figure 4 also applies to the relativistic FC method for solving the $\mathrm{DE}^{8}{ }^{8}$ with a small modification in that we use the inverse variational method ${ }^{24}$ to circumvent the variational collapse problem. The small and large components are correctly balanced automatically using the FC method. ${ }^{8}$

\section{Variational Calculations of the Ground and Excited States}

Applying the VP to the FC wave function given by eq 17 , we obtain the secular equation

$$
(\mathbf{H}-E \mathbf{S}) \mathbf{C}=\mathbf{0}
$$

where the Hamiltonian and overlap matrix elements, $H_{i j}$ and $S_{i j}$, respectively, are given by

$$
H_{i j}=\int \phi_{i} H \phi_{j} \mathrm{~d} \tau, \quad S_{i j}=\int \phi_{i} \phi_{j} \mathrm{~d} \tau
$$

Analytical integrations are possible for a system with few electrons, and below we briefly describe the results for a helium atom, $\mathrm{H}_{2}{ }^{+}$, and $\mathrm{H}_{2}$.

The Helium Atom in Its Ground and Excited States. Table 1 shows the VP results for a helium atom calculated by Nakashima $^{25}$ of our Institute. The FC method gives the energy in a chemical accuracy (three digits) in the first order and in a spectroscopic accuracy (six digits) in the second order. By continuing the calculations, we obtained the energy correct to 42 digits $^{25}$ and even to 45 digits $^{26}$ for the variational upper-bound energy. Schwartz also obtained a highly accurate energy assuming some functional form by intuition. ${ }^{27}$ We could calculate the lower-bound energy correct to 32 digits. $^{28}$ Therefore, the value shown in Table 1 is absolutely exact up to 32 digits. Thus, in principle, using 
TABLE 1. Ground-State Energy of the Helium Atom Calculated Using the FC-VP Method

\begin{tabular}{|c|c|c|c|}
\hline order $n$ & $M_{n}{ }^{a}$ & $\alpha$-opt $^{b}$ & energy $^{c}(\mathrm{au})$ \\
\hline 0 & 2 & 1.827 & $-\mathbf{2 . 8 6 5 3 7 0 8 1 9 0 2 6 7 1}$ \\
\hline 1 & 10 & 1.475 & $-\mathbf{2 . 9 0 3 5 3 6 8 1 2 2 8 1 5 3}$ \\
\hline 2 & 34 & 1.627 & $-\mathbf{2 . 9 0 3 7 2 4 0 0 7 3 2 1 4 5}$ \\
\hline 4 & 146 & 1.683 & -2.90372437702234 \\
\hline 26 & 20386 & $(2.17)$ & -2.90372437703411959831115924519440444669684021 \\
\hline 27 & 22709 & $(2.20)$ & -2.90372437703411959831115924519440444669690537 \\
\hline Schwartz & 10259 & & -2.9037243770341195983111592451944044400495 \\
\hline
\end{tabular}

${ }^{\bar{a}}$ Number of complement functions at order $n .{ }^{b}$ Optimal exponent. ${ }^{c}$ Correct figures are shown in bold face.

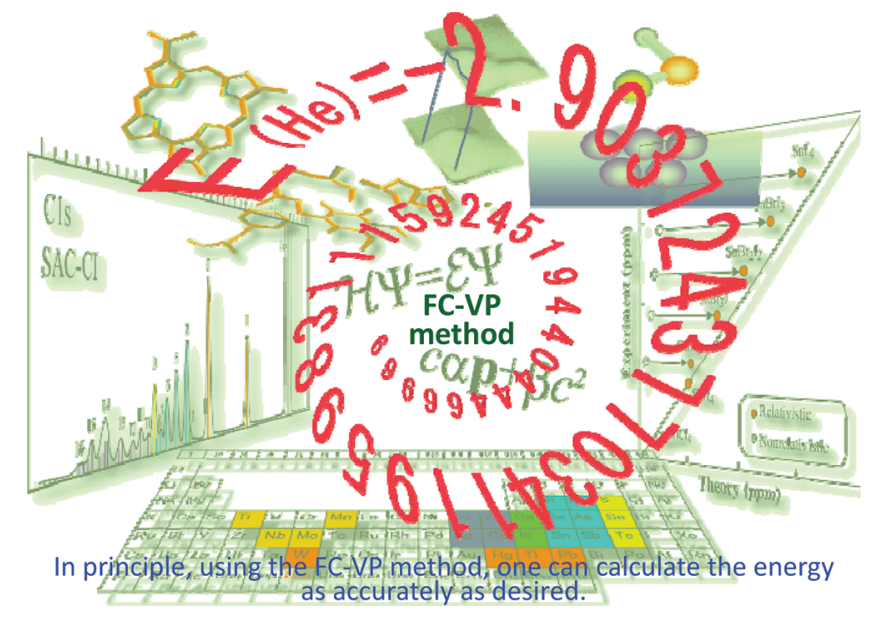

FIGURE 5. Energy of the helium atom calculated with the FC-VP method.

the FC-VP method, one can calculate the energy as accurately as desired, as illustrated in Figure 5 .

The calculations of the excited states are also interesting. Drake and Yan reported highly accurate energies of the excited states using a double basis set method. ${ }^{29}$ Table 2 shows the $1 \mathrm{~s}$ to $n$ s excitation energy of a helium atom calculated using the FC method at the nonrelativistic limit of the SE in a moving nucleus (non-BO) treatment. ${ }^{30}$ These results were obtained using a $\psi_{0}$ that was equally good for the calculation of both the ground and excited states. The bold numbers in the second column in Table 2 are the confidently correct numbers. The third column gives the experimental excitation energy, and the final column shows the difference between theory and experiment. Our results reproduce the experimental values to five digits. This degree of accuracy is similar to the exact case of the hydrogen atom explained earlier. Therefore, what do the values in the final column in Table 2 mean? Are they theoretical errors? No, they are not. As seen in the first column, our results are reliable to about 18 digits, giving a nonrelativistic limit. Therefore, the differences from the experimental values are mainly from relativistic effects, which are not included in our calculations. We can further point out the errors in the experimental values.
TABLE 2. Singlet $1 \mathrm{~s}$ to $n$ s Excitation Energies (au) of Helium Atom at the Nonrelativistic SE Level Compared with the Experimental Values

\begin{tabular}{|c|c|c|c|}
\hline state & FC-VP theory $^{a}$ & exptl. & $\Delta^{b}$ \\
\hline 2 & 0.757625970148997 & 0.7576157626 & 0.0000102075 \\
\hline 3 & 0.842315475380549 & 0.8423061388 & 0.0000093366 \\
\hline 4 & 0.869996740248283 & 0.8699881582 & 0.0000085821 \\
\hline 5 & 0.882404831556033 & 0.8823963512 & 0.0000084803 \\
\hline 6 & 0.889017646545153 & 0.8890092212 & 0.0000084253 \\
\hline 7 & 0.892954413277796 & 0.8929460170 & 0.0000083963 \\
\hline 8 & 0.895486211526316 & 0.8954778303 & 0.0000083812 \\
\hline 9 & 0.897210038952971 & 0.89720155 & 0.0000084869 \\
\hline 10 & 0.898436428853742 & 0.89842807 & 0.0000083569 \\
\hline 11 & 0.899339879169496 & 0.89933146 & 0.0000084226 \\
\hline 12 & 0.900024574123712 & 0.90001600 & 0.0000085738 \\
\hline 13 & 0.900555835611209 & 0.90054772 & 0.0000081109 \\
\hline 14 & 0.900976305419445 & 0.90096823 & 0.0000080766 \\
\hline 15 & 0.901314778505870 & 0.90130667 & 0.0000081051 \\
\hline$\infty$ & 0.903578706856692 & 0.903569891 & 0.0000088158 \\
\hline
\end{tabular}

In the $1 \mathrm{~s}$ to $n \mathrm{~s}$ series of excitations, the dependence on $n$ must be monotonic, but in the seventh digit, the change is not monotonic, which means that the experimental error begins at the seventh digit. Therefore, we conclude that numbers higher than the eighth digit in the experimental values are unreliable. Since the theory is accurate to 18 digits as the solution of the $\mathrm{SE}$, we can use it to predict outcomes. This is a small example of the role of "accurately predictive quantum chemistry".

Figure 6 shows a comparison of the ground and first excited states of a helium atom calculated using a nonrelativistic $S E$ and a relativistic $D E$ for a fixed nucleus to a moving nucleus treatment. The SE results are the same as those given in Table 1. In the nonrelativistic SE case, the theoretical value approaches the exact value from above, but in the relativistic case, the theoretical value approaches the exact value from below. The relativistic results always give better agreement with the experimental values. There are still small differences between the full relativistic $D E$ results and the experimental values, which are attributed to QED effects. Solving the SE and DE exactly is important, as this gives a well-defined starting point for further studies of QED and related effects. 


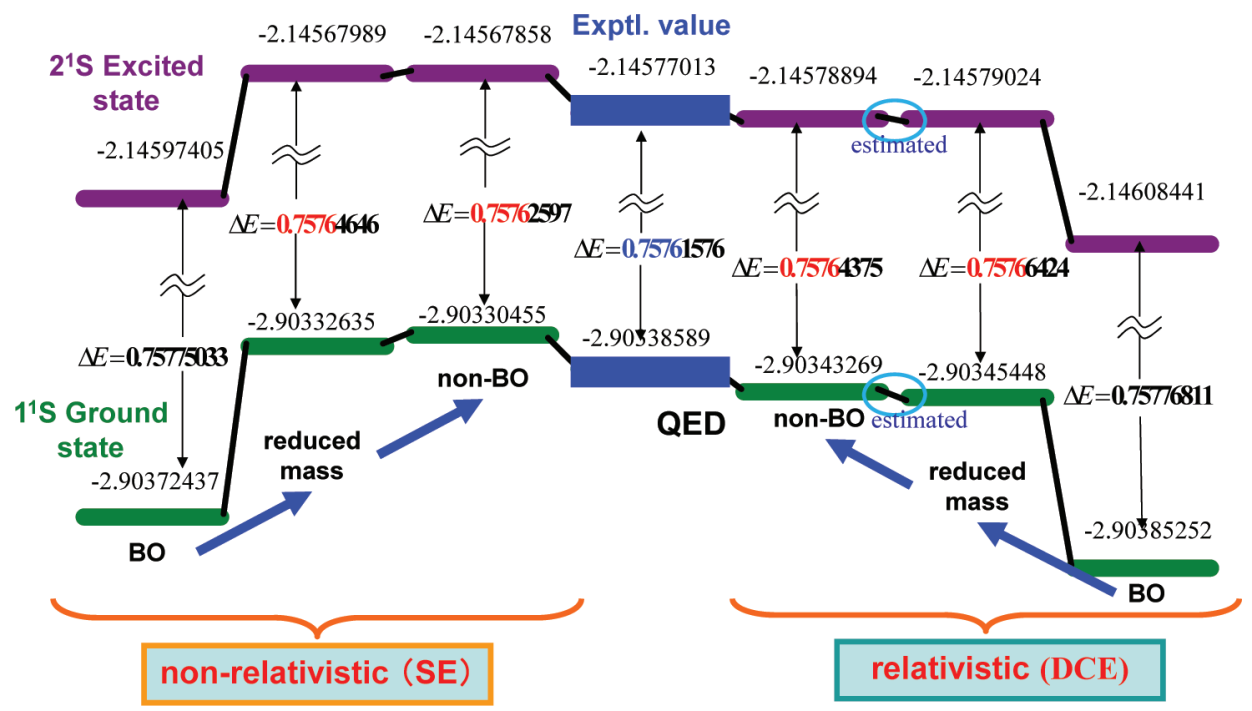

FIGURE 6. Ground and first excited states of the helium atom solved using the SE and DE.

TABLE 3. Results of the FC-LSE Calculations of Small Atoms and Molecules (au)

\begin{tabular}{|c|c|c|c|c|c|c|}
\hline system & no. of electrons & $n$ & $M$ & FC-LSE energy (au) & best ref energy (au) & $\Delta E^{a}(\mathrm{kcal} / \mathrm{mol})$ \\
\hline $\mathrm{He}$ & 2 & 6 & 100 & -2.90372452 & -2.90372437 & -0.000094 \\
\hline $\mathrm{Li}^{+}$ & 2 & 6 & 100 & -7.27991433 & -7.27991341 & -0.00058 \\
\hline $\mathrm{H}_{2}$ & 2 & 5 & 388 & -1.17447477 & -1.17447593 & 0.00075 \\
\hline $\mathrm{Li}$ & 3 & 6 & 1496 & -7.478043 & -7.478060 & 0.011 \\
\hline $\mathrm{Be}^{+}$ & 3 & 6 & 1496 & -14.324753 & -14.324763 & 0.006 \\
\hline $\mathrm{He}_{2}{ }^{+}$ & 3 & 6 & 2632 & -4.994646 & -4.994644 & -0.0012 \\
\hline $\mathrm{Be}$ & 4 & 4 & 1770 & -14.667300 & -14.667355 & 0.034 \\
\hline $\mathrm{B}$ & 5 & 4 & 15038 & -24.653872 & -24.65393 & 0.036 \\
\hline
\end{tabular}

$\mathbf{H}_{\mathbf{2}}{ }^{+}$and $\mathbf{H}_{2} \cdot \mathrm{H}_{2}{ }^{+}$is a unique molecule, for which the exact solution of the SE is known. ${ }^{23}$ We applied the FC method and compared it with the exact method and found that the FC method converges more rapidly than the exact method. ${ }^{23}$

$\mathrm{H}_{2}$ is a landmark molecule with which Heitler and London $^{31}$ initiated quantum chemistry in 1927. There is much history on the study of this molecule, and it was also the first molecule to which the FC method was applied. ${ }^{32}$ We found that the FC wave function that was generated automatically with the Hamiltonian was different from the existing wave functions, and this difference was shown to be physically important. The calculated result was the best variationally calculated result at that time, though later on, a better wave function was obtained by Sims and Hagstrom. ${ }^{33}$

\section{Integration-Free Solution: Local SE Method}

When the VP method is applicable, we can obtain very accurate results almost automatically, as shown above. However, this is not always possible, because in general, analytical integrations over the complement functions given by eq 19 are difficult and time-consuming. The FC method does not care about the integratability of the complement functions produced. This difficulty is referred to as the integration difficulty. ${ }^{10}$

However, the potential exactness of our FC wave function provided a hint on how to overcome this difficulty. Since the FC wave function of eq 17 has an exact structure, the coefficients involved should be determined using only the necessary conditions of the SE. We use a local SE (LSE),

$$
H \psi\left(r_{\mu}\right)=E \psi\left(r_{\mu}\right)
$$

at different coordinates $r_{\mu}$ as such a necessary condition. When we introduce the local energy $\varepsilon_{\mathrm{L}}\left(r_{\mu}\right)=H \psi\left(r_{\mu}\right) / \psi\left(r_{\mu}\right)$, the $S E$ requires the local energy to be flat everywhere at $E$. This flatness was clearly depicted using the FC wave function of the He atom. ${ }^{28}$ For the FC wave function given by eq 17 , we can rewrite eq 20 as

$$
\mathbf{A c}=E \mathbf{B c}
$$


TABLE 4. Comparison of Full-Cl and FC-LSE Calculations for Be and $\mathrm{LiH}^{a}$

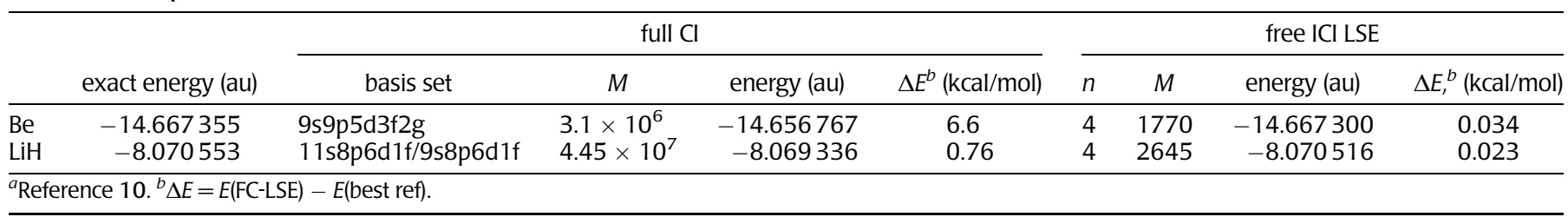

TABLE 5. FC-LSE Calculations of 5-12 Electron Atoms

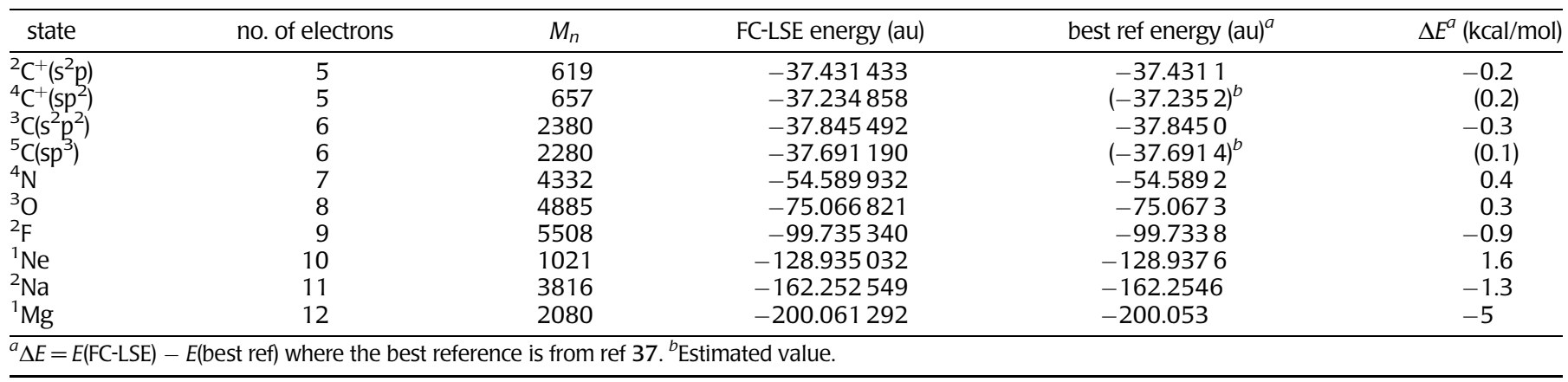

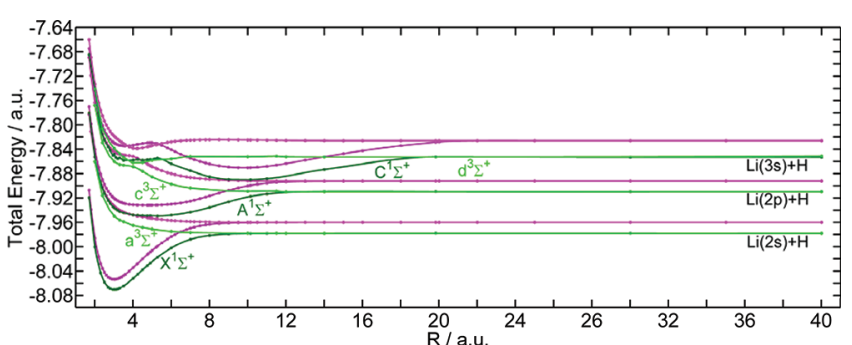

FIGURE 7. Lowest three ${ }^{1,3} \Sigma^{+}$potential energy curves of LiH calculated by the FC-LSE method (green) and the full-Cl/ccp-V5Z method (magenta) for interatomic distances between 1.7 and 40.0 au.

where $\left\{A_{\mu i}\right\}=\left\{H \phi_{i}\left(r_{\mu}\right)\right\},\left\{B_{\mu i}\right\}=\left\{\phi_{i}\left(r_{\mu}\right)\right\}$, and $\mathbf{c}$ is a column vector of $\left\{\boldsymbol{c}_{i}\right\}$. The matrices $\mathbf{A}$ and $\mathbf{B}$ are highly nonsymmetric. This method assumes the flatness of the local energy evenly in the set of sampled points, $r_{\mu}$. The ground state is the lowest solution, and the excited states are the higher solutions. The LSE method is similar in spirit to the least-squares local-energy method considered by Frost many years ago. ${ }^{34}$

When we multiply $\mathbf{B}^{\mathrm{T}}$ from the left, we obtain

$$
\mathbf{H c}=E \mathbf{S c}
$$

where $S_{i j}=\Sigma_{\mu} \phi_{i}\left(r_{\mu}\right) \phi_{j}\left(r_{\mu}\right)$ and $\mathbf{S}$ is symmetric and positive definite. At the integral limit, it becomes the overlap integral between the complement functions $\phi_{i}$ and $\phi_{j}$. Similarly, $H_{i j}=\Sigma_{\mu} \phi_{i}\left(r_{\mu}\right) H \phi_{j}\left(r_{\mu}\right)$, which is close to the Hamiltonian matrix element but is nonsymmetric. On increasing the number of sampling points, the results should
TABLE 6. FC-LSE Structural and Vibrational Properties of LiH Compared with Experimental Values

\begin{tabular}{lrrrrrr}
\hline & $r_{\mathrm{e}}, \mathrm{au}$ & $G_{0}, \mathrm{~cm}^{-1}$ & $\Delta G_{0}, \mathrm{~cm}^{-1}$ & $\Delta G_{1}, \mathrm{~cm}^{-1}$ & $D_{\mathrm{e},} \mathrm{eV}$ & $T_{\mathrm{e},} \mathrm{eV}$ \\
\hline & \multicolumn{5}{c}{ X State } \\
FC-LSE & 3.013 & 695.41 & 1356.87 & 1312.79 & 2.521 & \\
exptl & 3.015 & 697.94 & 1359.71 & 1314.89 & 2.515 & \\
& & & A State & & & \\
FC-LSE & 5.130 & 136.05 & 298.42 & 301.57 & 1.075 & 3.296 \\
exptl & 4.906 & 131.30 & 280.84 & 312.97 & 1.076 & 3.272 \\
\hline
\end{tabular}

become closer to the variational results. The Monte Carlo method is one choice for sampling, but to prevent its random behavior, we proposed the local transferable sampling method. ${ }^{35}$ This gives continuous results that are favorable for drawing potential curves and for calculating potential energy derivatives, such as vibrational frequencies.

As far as we use the LSE method, any complement function is acceptable, since we do not calculate their integrals. Therefore, it is interesting to try many different analytical functions for $\psi_{0}$ that produce complement functions by applying the Hamiltonian.

\section{FC-LSE Calculations of Atoms and Molecules}

Table 3 shows the results of LSE calculations for small atoms and molecules. ${ }^{10}$ We used a $10^{7}$ Monte Carlo sampling. Though these systems are small, the calculated energies are highly accurate. The deviations from the known exact energies are less than $0.04 \mathrm{kcal} / \mathrm{mol}$. In Table 4, we compare 
the FC-LSE results for Be and $\mathrm{LiH}$ with the full-Cl results. ${ }^{10}$ Though these full-Cl results used a highly accurate basis set, the error still amounted to a few $\mathrm{kcal} / \mathrm{mol}$, but in our method, the error was about $0.03 \mathrm{kcal} / \mathrm{mol}$. Table 5 shows the results for atoms with 5-12 electrons. Because carbon is especially important, we calculated different electronic states for it. Up to fluorine, we could obtain chemical accuracy, where the error was less than a $\mathrm{kcal} / \mathrm{mol}$. For $\mathrm{Ne}$ to $\mathrm{Mg}$, the errors were larger, though it was not difficult to obtain higher accuracies. Thus, without carrying out any integration, we could calculate highly accurate energies and wave functions in an analytical expansion form.

\section{Potential Energy Curves}

Potential energy curves and surfaces are of fundamental importance in chemical studies. Here, we show an example of the potential curves of the $\mathrm{LiH}$ molecule ${ }^{36}$ in Figure 7. The lowest two singlet and triplet $\Sigma^{+}$potential curves were calculated using the FC-LSE method (green curves), and these can be compared with those obtained from full-Cl calculations using the cc-pV5Z basis set (magenta). The FC-LSE curves and the full-Cl curves are parallel in all the regions, although the FC-LSE curves are always lower in energy than the full-Cl curves. The potential properties are summarized in Table 6 and compared with the experimental values. The equilibrium distance, $r_{\mathrm{e}}$, zero-point vibrational energy, $G_{0}$, vibrational level spacing, $\Delta G_{0} \Delta G_{1}$, the dissociation energy, $D_{\mathrm{e}}$, and the adiabatic excitation energy between two minima, $T_{\mathrm{e}}$, show good accordance between theory and experiment. The accordance is lower in the excited state, but the very shallow nature of the potential curve leads to a fluctuation in the fitted values. These results show the utility of the FC-LSE method for study of chemical reactions in ground and excited states.

\section{Petaflops Superparallel Computing}

In recent developments in computer technology, superparallel computers with petaflops to exaflops speeds are being produced. For most conventional quantum chemistry codes, the adaptation to superparallel computation is not an easy task. However, for the FC-LSE method, the most timeconsuming step is the sampling step (calculation of the values of $H \phi_{i}\left(r_{\mu}\right)$ and $\phi_{i}\left(r_{\mu}\right)$ in eq 21 for each sampling point $r_{\mu}$ ). Because we usually take $10^{5}-10^{7}$ sampling points in our calculations, and each sampling point has exactly the same computational task, superparallel computers are suitable for FC-LSE calculations. In test calculations on $\mathrm{N}_{2}$ molecules, we used 1152 cores in parallel and obtained an acceleration of 1150 times (99.8\% parallel efficiency). We are looking forward to the open usage of the petaflops computers at Kobe.

\section{Present and Future}

After the discovery of the general theory of solving the SE, we have investigated and developed different methods and algorithms in order to apply this general theory to atoms and molecules of different sizes and in different situations. The LSE method is one example that forced us to develop a sampling-type methodology. The methods described here are general, and are therefore, in principle, applicable to any system. However, the algorithms we have reviewed here are the straightforward ones derived from the original theories. They are still time-consuming to apply to molecules familiar in everyday chemistry, and it is therefore important to invent more efficient methods. We are now developing several ideas in parallel. Further, with the active research of many scientists who are interested in this subject, we hope this field is much cultivated and will offer innovative methodologies that make confident quantitative predictions possible in the field of chemical science.

I acknowledge Dr. Hiroyuki Nakashima of QCRI for active collaborations.

\section{BIOGRAPHICAL INFORMATION}

Hiroshi Nakatsuji has been the Director of the Quantum Chemistry Research Institute since 2006 and a Professor Emeritus of Kyoto University since 2007. He is the author of the force concept for molecular geometries and chemical reactions, the density equation for the direct determination of density matrix, the SAC and SAC-Cl theories for ground, excited, ionized, and electronattached states of molecules, the dipped adcluster model for chemisorption and catalytic reactions on a metal surface, and studies on the mechanisms and relativistic effects in nuclear magnetic resonance.

\section{FOOTNOTES}

*E-mail: h.nakatsuji@qcri.or.jp.

The authors declare no competing financial interest.

\section{REFERENCES}

1 Dirac, P. A. M. Quantum mechanics of many-electron systems. Proc. R. Soc. London, Ser. A 1929, 123, 714-733.

2 Hawking, S.; Mlodinow, L. The Grand Design; Bantam Books: London, 2010.

3 Piecuch, P.; Kucharski, S. A.; Kowalski, K. Can ordinary single-reference coupled-cluster methods describe the potential curve of $\mathrm{N}_{2}$ ? The renormalized $\operatorname{CCSDT}(\mathrm{Q})$ study. Chem. Phys. Lett. 2001, 344, 176-184.

4 Nakatsuji, H. Structure of the exact wave function. J. Chem. Phys. 2000, 113, 29492956. 
5 Nakatsuji, H.; Davidson, E. R. Structure of the exact wave function. II. Iterative configuration interaction method. J. Chem. Phys. 2001, 115, 2000-2006.

6 Nakatsuij, H. Inverse Schrödinger equation and the exact wave function. Phys. Rev. A 2002, 65, №. 052122

7 Nakatsuji, H. Scaled Schrödinger equation and the exact wave function. Phys. Rev. Lett. 2004, 93, No. 030403

8 Nakatsuji, H. General method of solving the Schrödinger equation of atoms and molecules. Phys. Rev. A 2005, 72, No. 062110.

9 Nakatsuji, H.; Nakashima, H. Analytically solving relativistic Dirac-Coulomb equation for atoms and molecules. Phys. Rev. Lett. 2005, 95, No. 050407.

10 Nakatsuji, H.; Nakashima, H.; Kurokawa, Y.; Ishikawa, A. Solving the Schrödinger equation of atoms and molecules without analytical integration based on the free iterativecomplement- interaction wave function. Phys. Rev. Lett. 2007, 99, №. 240402.

11 Sakurai, J. J. Advanced Quantum Mechanics; Addison-Wesley: CA, 1967.

12 Moore, C. E. Atomic Energy Levels; National Bureau of Standards: Gaithersburg, MD, 1971; Vol. I.

13 Nakatsuji, H. Structure of the exact wave function. III. Exponential ansatz. J. Chem. Phys. $2001,115,2465-2475$

14 Nakatsuji, H. Structure of the exact wave function. IV. Excited states from exponential ansatz and comparative calculations by the iterative configuration interaction and extended coupled cluster theories. J. Chem. Phys. 2002, 116, 1811-1824.

15 Nakatsuji, H.; Ehara, M. Structure of the exact wave function. V. Iterative configuration interaction method for molecular systems within finite basis. J. Chem. Phys. 2002, 117, 9-12.

16 Nakatsuij, H. Equation for the direct determination of the density matrix. Phys. Rev. A 1976 $14,41-50$.

17 Nakatsuji, H. Deepening and extending the quantum principles in chemistry. Bull. Chem. Soc. Jpn. 2005, 78, 1705-1724.

18 Nakatsuij, H. Full configuration-interaction calculations with the simplest iterative complement method: Merit of the inverse Hamiltonian. Phys. Rev. A. 2011, 84, No. 062507.

19 Nakatsuji, H.; Ehara, M. Iterative $\mathrm{Cl}$ general singles and doubles (ICIGSD) method for calculating the exact wave functions of the ground and excited states of molecules. J. Chem. Phys. 2005, 122, No. 194108.

20 Nakatsuji, H. Local g operator in the formation of the complement functions. Manuscript in preparation.

21 Nakatsuji, H.; Nakashima, H. How does the free complement wave function become accurate and exact finally for the hydrogen atom starting from the Slater and Gaussian initial functions and for the helium atom on the cusp conditions? Intern. J. Quantum Chem. 2009, 109, 2248-2262.
22 Nakashima, H.; Nakatsuij, H. Solving the Schrödinger and Dirac equations for a hydrogen atom in the universe's strongest magnetic fields with the free complement method. Astrophys. J. 2010, 725, 528-533.

23 Ishikawa, A.; Nakashima, H.; Nakatsuji, H. Accurate solutions of the Schrödinger and Dirac equations of $\mathrm{H}_{2}{ }^{+}, \mathrm{HD}^{+}$, and $\mathrm{HT}^{+}$: With and without Born-Oppenheimer approximation and under magnetic field. Chem. Phys. 2012, 401, 62-72.

24 Hill, R. N.; Krauthauser, C. A solution to the problem of variational collapse for the oneparticle Dirac equation. Phys. Rev. Lett. 1994, 72, 2151-2154.

25 Nakashima, H.; Nakatsuji, H. Solving the Schrödinger equation for helium atom and its isoelectronic ions with the free iterative complement interaction (ICI) method. J. Chem. Phys. 2007, 127, №. 224104

26 Kurokawa, Y.; Nakashima, H.; Nakatsuij, H. Solving the Schrödinger equation of helium and its isoelectronic ions with the exponential integral (Ei) function in the free iterative complement interaction method. Phys. Chem. Chem. Phys. 2008, 10, 4486-4494.

27 Schwartz, C. Experiment and theory in computations of the He atom ground state. Int. J. Mod. Phys. E 2006, 15, 877-888.

28 Nakashima, H.; Nakatsuji, H. How accurately does the free complement wave function of a helium atom satisfy the Schrödinger equation? Phys. Rev. Lett. 2008, 101, №. 240406.

29 Drake, G. W. F.; Van, Z.-C. Variational eigenvalues for the S states of helium. Chem. Phys. Lett. 1994, 229, 486-490.

30 Nakashima, H.; Hijikata, Y.; Nakatsuji, H. Solving the electron and electron-nuclear Schrödinger equations for the excited states of helium atom with the free iterativecomplement-interaction method. J. Chem. Phys. 2008, 128, №. 154108.

31 Heitler, W.; London, F. Wechselwirkung neutraler atome und homöopolare bindung nach der quantenmechanik. Z. Phys. A 1927, 44, 455-472.

32 Kurokawa, Y.; Nakashima, H.; Nakatsuji, H. Free ICI (Iterative Complement Interaction) calculations of the hydrogen molecule. Phys. Rev. A 2005, 72, NNo. 062502.

33 Sims, J. S.; Hagstrom, S. A. High precision variational calculations for the BornOppenheimer energies of the ground state of the hydrogen molecule. J. Chem. Phys. 2006, 124, №. 094101.

34 Frost, A. A.; Kellogg, R. E.; Gimarc, B. M.; Scargle, J. D. Least-square local-energy method for molecular calculations using Gauss quadrature points. J. Chem. Phys. 1961, 35, 827-831.

35 Nakatsuij, H. Local sampling method that is transferrable and gives continuity to the calculated properties. Manuscript in preparation.

36 Bande, A.; Nakashima, H.; Nakatsuji, H. LiH potential energy curves for ground and excited states with the free complement local Schrodinger equation method. Chem. Phys. Lett. 2010, 496, 347-350.

37 Buendia, E.; Galvez, F. J.; Maldonado, P.; Sarsa, A. Quantum Monte Carlo ground state energies for the atoms Li through Ar. J. Chem. Phys. 2009, 131, №. 044115. 